\title{
Short Review on Sublingual Immunotherapy for Patients with Allergic Rhinitis: from Bench to Bedside
}

\author{
Hideyuki Kawauchi · Kaoru Goda • Miki Tongu • Takaya Yamada • \\ Noriaki Aoi • Ichiro Morikura · Takashi Fuchiwaki \\ Department of Otorhinolaryngology, Shimane University, Faculty of Medicine, Izumo, Japan
}

\begin{abstract}
Sublingual immunotherapy has been considered to be a painless and effective therapeutic treatment for allergic rhinitis, and is known as type 1 allergy of the nasal mucosa. So far, its mechanism of action has been elucidated employing peripheral blood serum and lymphocytes in an antigen-specific fashion. Because of the limitations in sampling human materials, there is still controversy among many reports between clinical efficacy and laboratory data. Therefore, its mechanism of action needs to be investigated further by using promising animal models such as rodents and monkeys. Bearing this in mind, in our present study, we successfully constructed an effective murine model for sublingual immunotherapy in allergic rhinitis in which mice were administered ovalbumin (OVA) sublingually followed by intraperitoneal sensitization and nasal challenge.
\end{abstract}

Copyright @ 2011 S. Karger AG, Base

\section{Allergic Rhinitis and Regulatory Mechanism for Allergic Inflammation}

Allergic rhinitis is one of the commonest types of allergic inflammation affecting $10-25 \%$ of the world's population, and its prevalence has increased over the last decade. Allergic rhinitis is characterized by a predominant Th2 response, antigen-specific IgE synthesis and infiltration of eosinophils into the nasal mucosa [1]. The type 1 allergic response is largely divided into two phases, namely systemic response at the induction phase and allergic inflammation in the eliciting phase. The eliciting phase is further divided into two phases, namely immediate phase and late phase. To consider the pathologies of type 1 allergic inflammation, we should now distinguish between the induction phase and the eliciting phase, even though there is always a mixture of these two phases in normal human nasal mucosa. The hygiene hypothesis in line with helper $\mathrm{T}$ cell dichotomy has been proposed by a large number of investigators and many plausible hypotheses are available to understand allergic inflammation in comparison with infective inflammation.

More recently, regulatory $\mathrm{T}$ cells, defined as CD4+CD25+ FoxP3+ T cell subpopulations, have been focused on as key players to downregulate excessive Th1 or Th2 pathologies such as autoimmune disease or allergy. These regulatory $\mathrm{T}$ cell subpopulations are considered to be efficiently 
developed in a circumstance of low hygiene and high pathogen/adjuvant burden. Regulatory $\mathrm{T}$ cells producing IL-10 and/or TGF- $\beta$ are induced not only in atopic patients by successful immunotherapy, but also during natural allergen exposure in healthy people.

Regulatory $\mathrm{T}$ cells can control and regulate all the effector mechanisms activated during allergy and the Th2 responses through the production of IL-10/TGF- $\beta$ and/or cell-cell contact. IL-10 is a potent suppressor of total and allergenspecific IgEs, whereas it induces an antibody isotype switch towards IgG4. TGF- $\beta$ also decreases IgE production and induces an immunoglobulin isotype switch towards IgA. IL-10 and TGF- $\beta$ act directly or indirectly on the human airways to decrease both mucus production and airway hyperreactivity.

\section{Antigen-Specific Subcutaneous - and Sublingual - Immunotherapy}

In the 5 th edition of Japanese guidelines of allergic rhinitis, specific immunotherapy is recommended and introduced. So we need a more elegant and safe immunotherapy rather than the conventional one, and now sublingual immunotherapy has been in trials with $\mathrm{T}$ cell epitopes of cedar pollen in patients suffering from cedar pollenosis. Peptide immunotherapy with the T cell epitope of allergens (percutaneously or sublingually) is now in the process of clinical trials in Japan.

For the better understanding of sublingual immunotherapy, we need information such as standardization of antigens for immunotherapy, clinical efficacy, safety, mechanism of action, and aim for early intervention. Allergen-specific immunotherapy is associated with an improved tolerance to allergen challenge, with a decrease in immediate- and late-phase allergic inflammation [2]. Allergen-specific immunotherapy also modifies cellular and humoral responses to the allergen. The ratio of Th 1 cytokines to Th2 cytokines is increased following allergen-specific immunotherapy, and functional regulatory $\mathrm{T}$ cells are induced. Production of blocking antibodies, IgG1, $\operatorname{IgG} 4$ and $\operatorname{IgA}$, is induced and these immunoglobulins compete with IgE for allergen binding, thereby decreasing the allergen capture and presentation that is facilitated by the IgE in complex with FceRI or FceRII.

Sublingual immunotherapy has previously been shown in many clinical studies to modulate allergen-specific $\mathrm{Th} 2$ responses with a decrease in the IgE to IgG4 ratio, and its mechanism has been considered similar to that of subcutaneous immunotherapy [3-7]. Here, we address some new findings of the immunological mechanisms of sublingual immunotherapy such as decrease in the IgE/IgG4 ratio, induction of IL10-producing Tregs and allergen-specific $\mathrm{T}$ cell tolerance, etc. Cosmi's group have performed a clinical long-term study for sublingual immunotherapy in patients with perennial rhinitis with Dermatophagoides and came to the conclusion that the effects of SLIT is downregulation of Dpspecific IgE, enhancement of IFN- $\gamma$ with Th cells, downregulation of Dp-specific proliferation, and enhancement of IL-10 production. On the other hand, in their clinical study of patients with birch pollenosis, Bohler et al. [8] found that transient induction of regulatory $\mathrm{T}$ cells immediately after SLIT is followed by long-term allergen-specific peripheral tolerance (anergy) and immune deviation (from Th2 to Th1 milieu). Then, why do we choose sublingual immunotherapy as a new therapeutic method rather than subcutaneous immunotherapy?

Sublingual immunotherapy requires much more amounts of allergen than subcutaneous immunotherapy, but it has several benefits in comparison with subcutaneous immunotherapy [9]. Sublingual immunotherapy is pain-free and safer than subcutaneous immunotherapy, because the amount of mast cells is least in the sublingual mucosal tissue. Another benefit is that patients can carry out the immunotherapy procedure by 


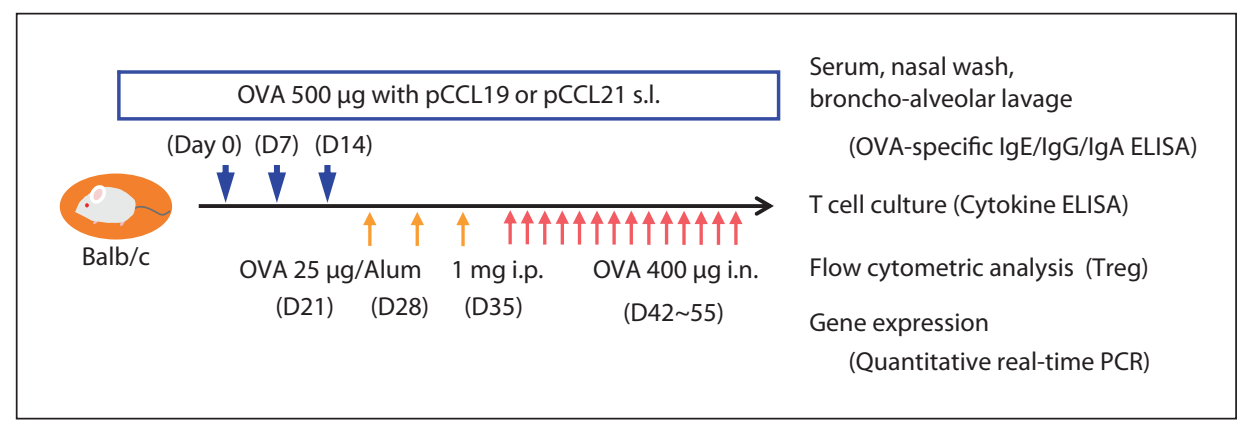

Fig. 1. Induction of allergic rhinitis and sublingual immunotherapy: Mice were sensitized by intraperitoneal injections (i.p.) of $25 \mu \mathrm{g}$ of OVA with $1 \mathrm{mg}$ of alum every week for three times and were nasally challenged (i.n.) with $400 \mu \mathrm{g}$ of OVA for 14 consecutive days. For sublingual treatment (s.l.), $4 \mu \mathrm{l}$ of PBS alone or PBS containing $500 \mu \mathrm{g}$ of OVA was applied on the sublingual mucosa of anesthetized mice before sensitization. Mice and specimens: 6- to 8-week-old Balb/c mice were used. After the final nasal challenge, serum, nasal wash and broncho-alveolar lavage (BAL) were collected. Single cells of spleen, cervical lymph node (CLN), nasopharynx-associated lymphoid tissue (NALT), and nasal passage were harvested from the mice.

themselves at home after reaching the allergen maintenance dose, thus necessitating fewer visits to the outpatient clinic. To solve the problem of sublingual immunotherapy requiring a high dose of allergen, improvement of the allergen delivery system is necessary. One possible way is to modify immunocompetent cells such as dendritic cells for the efficient induction of regulatory $\mathrm{T}$ cells and the production of allergen-specific IgGs.

\section{Experimental Data in Our Murine Allergic Rhinitis Model and Future Goal}

Sublingual immunotherapy has been considered to be a painless and effective therapeutic treatment for allergic rhinitis, which is a known as type 1 allergy of the nasal mucosa. So far, its mechanism of action has been elucidated by employing peripheral blood serum and lymphocytes in an antigen-specific fashion. Controversy still exists between many reports regarding the clinical efficacy and laboratory data of this method because of the limitations in sampling human material. Therefore, its mechanism of action needs to be further investigated using promising animal models such as rodents and monkeys. Bearing this in mind, in our present study, we successfully constructed an effective murine model for sublingual immunotherapy in allergic rhinitis in which mice were administered ovalbumin (OVA) sublingually followed by intraperitoneal sensitization and nasal challenge. The materials and methods are shown in detail in figure 1. To summarize our experimental data, sublingually treated mice showed significantly decreased allergic responses as well as suppressed Th2 immune responses and sublingual administration of OVA did not alter the frequency of CD4+CD25+ regulatory $\mathrm{T}$ cells (Tregs), but led to upregulation of Foxp3- and IL-10-specific mRNAs in the Tregs of cervical lymph nodes (CLN). These results suggest that IL-10 expressing CD4+CD25+Foxp3+ Tregs in CLN are involved in the suppression of allergic responses [10]. 


\section{Conclusion}

The future goal, from the research and clinical aspects, is that antigen dosing via the sublingual route should be precisely examined with $\mathrm{T}$ cell epitopes to increase clinical efficacy and avoid adverse events. Different types of antigen delivery systems should be developed to replace the conventional methods.

\section{Acknowledgement}

This study was supported by Grant-in-Aids from the Japanese Ministry of Education, and Agriculture.

\section{References}

1 Gelfand EW: Inflammatory mediators in allergic rhinitis. J Allergy Clin Immunol 2004;114:S135-S138.

2 Larche M, Akdis CA, Valenta R: Immunological mechanisms of allergenspecific immunotherapy. Nat Rev Immunol 2006;6:761-771.

- 3 Wilson DR, Torres Lima M, Durham SR: Sublingual immunotherapy for allergic rhinitis: systematic review and metaanalysis. Allergy 2005;60:4-12.

4 Song JH, Kim JI, Kwon HJ, et al: CCR7CCL19/CCL21-regulated dendritic cells are responsible for effectiveness of sublingual vaccination. J Immunol 2009; 182:6851-6860.
5 Ciprandi G, Fenoglio D, Cirillo I, et al: Sublingual immunotherapy: an update on immunologic and functional effect. Allergy Asthma Proc 2007;28:40-43.

6 Okubo K, Gotoh M, Fujieda S, et al: A randomized double-blind comparative study of sublingual immunotherapy for cedar pollinosis. Allergol Int 2008;57: 265-227.

7 Moingeon P, Batard T, Fadel R, et al: Immune mechanisms of allergenspecific sublingual immunotherapy. Allergy 2006;61:151-165.
8 Bohler B, et al: SLIT induces IL-10producing Tregs and allergen-specific T cell tolerance. J Allergy Clin Immunol 2007;120:707-713.

-9 Yamanaka K, Yuta A, Kakeda M, et al: Induction of IL-10-producing regulatory $\mathrm{T}$ cells with TCR diversity by epitopespecific immunotherapy in pollinosis. J Allergy Clin Immunol 2009;124: 842-845, e7.

10 Goda K, Kawauchi H, et al: An investigation of the mechanism of sublingual immunotherapy: an experimental data in murine allergic rhinitis model. Jpn J Stomatopharyngol 2009;22:31-33.

Dr. Hideyuki Kawauchi

Department of Otorhinolaryngology, Shimane University, Faculty of Medicine

89-1 Enya-cho

Izumo City, Shimane 693-8501 (Japan)

Tel. +81 85320 2273, E-Mail kawauchi@med.shimane-u.ac.jp 